\title{
Effective field investigation of dynamic phase transitions for site diluted Ising ferromagnets driven by a periodically oscillating magnetic field
}

\author{
U. Akinci, Y. Yuksel, E. Vatansever and H. Polat ${ }^{\dagger}$ \\ Deparment of Physics, Dokuz Eylul University, Buca/Izmir-35160, Turkey \\ E-mail: hamza.polat@deu.edu.tr
}

\begin{abstract}
Dynamic behavior of a site diluted Ising ferromagnet in the presence of periodically oscillating magnetic field has been analyzed by means of the effective field theory (EFT). Dynamic equation of motion have been solved for a honeycomb lattice $(z=3)$ with the help of a Glauber type stochastic process. The global phase diagrams and the variation of the corresponding dynamic order parameter as a function of the Hamiltonian parameters and temperature has been investigated in detail and it has been shown that the system exhibits reentrant phenomena, as well as a dynamic tricritical point which disappears for sufficiently weak dilution.
\end{abstract}




\section{Introduction}

Ising model under a time dependent external magnetic field (kinetic Ising model) has attracted much interest from both theoretical and experimental points of view recently. Due to the competing time scales of the system and periodic external magnetic field, the system cannot respond to the external magnetic field instantaneously which causes interesting behaviors. At high temperatures for the high amplitudes of the periodic magnetic field, the system follows the external field with a delay while this is not the case for low temperatures and small magnetic field amplitudes. This spontaneous symmetry breaking indicates the presence of a dynamical phase transition (DPT) [1] which shows itself in the dynamical order parameter (DOP) which is defined as the time average of the magnetization over a full period of the oscillating field. DPT is firstly observed theoretically within the mean field approximation (MFA) [2]. Since then, much attention has been devoted to investigate the dynamic nature of the phase transition in experimental studies. As an example, a DPT occurring in high frequency region is represented by Jiang et al. [3] using the surface magneto-optical Kerr-effect technique for epitaxially grown ultrathin Co films on a $\mathrm{Cu}(001)$ surface. For a $\left[\mathrm{Co}\left(4 \mathrm{~A}^{\circ}\right) / \mathrm{Pt}\left(7 \mathrm{~A}^{\circ}\right)\right]$ multilayer system with strong perpendicular anisotropy, an example of DPT is shown by Robb et al. 44. In this study, they found that the experimental nonequilibrium phase diagrams are found to strongly resemble the dynamic behavior calculated from simulations of a kinetic Ising model. Thus, a strong evidence of consistency between theoretical and experimental studies is shown. Polyethylene naphtalate nanocomposites are investigated by Kanuga et al. [5]. After detailed analysis, they shown that the material undergoes three critical structural transitions.

DPT and hysteresis behaviors of the S-1/2 Ising model with periodic external magnetic field has been studied by means of MFA [6, 7, 8, 9, 10] and Monte Carlo (MC) simulations [11, 12, 13, 14, 15, 16, 17], and there are also some works for higher spins [18, 19] or mixed spin Ising systems [20, 21], as well as the other variants of the model such as the transverse kinetic Ising model [22] based on the MFA.

It is well known that MFA neglects the thermal fluctuations via neglecting the self spin correlations whereas the effective field theory (EFT) which is superior to conventional MFA is based on the differential operator technique [23, 24] and it takes into account the self spin correlations. Thus, it is expected from EFT to obtain more reasonable results than MFA for these systems, as in the case of static Ising model. EFT has been applied to the kinetic Ising model in the past few years. For example, thermal and magnetic properties of kinetic Ising model [25, 26, 27, 28, and also the transverse kinetic Ising model [29, 30] have been studied by EFT very recently. Based on the results obtained in these works, we can conclude that EFT gives more reasonable results for the investigation of the DPT behaviors of the kinetic Ising model, in comparison to MFA.

On the other hand, as far as we know, the thermal and magnetic properties of the quenched disordered systems such as site (or bond) diluted ferromagnets driven by a periodically oscillating magnetic field have not yet been investigated within the 
framework of EFT or MFA . Furthermore, DPT properties of such disordered materials have not yet also been considered by means of MC simulations. These types of disorder effects constitute an important role in material science, since the quenched disorder effects may induce some important macroscopic effects on the material. Therefore, we believe that the investigation of quenched randomness effects on the DPT properties of Ising model still needs particular attention and we need a detailed consideration of competition between the disorder conditions and the oscillation of magnetic field. Hence, in this work we intend to study the DPT properties of a kinetic Ising system by introducing the quenched site dilution effects. For this purpose, we organized the paper as follows: In Sec. 2 we briefly present the formulations. The results and discussions are summarized in Sec. 3, and finally Sec. 4 contains our conclusions.

\section{Formulation}

We consider an Ising ferromagnet defined on a lattice which has a coordination number $z$ with a time dependent external magnetic field. The Hamiltonian describing our model is

$$
\mathcal{H}=-J \sum_{<i, j>} c_{i} c_{j} s_{i} s_{j}-H(t) \sum_{i} c_{i} s_{i}
$$

where $J>0$ is spin-spin exchange interaction, $c_{i}$ is a site occupation variable and $s_{i}$ is the spin variable. Site occupation variable can take the values $c_{i}=0$ which means that the site $i$ is empty or $c_{i}=1$ if the site $i$ is occupied by a magnetic atom and the spin variable can take values $s_{i}= \pm 1$. The first summation in Eq. (11) is over the nearest neighbor site pairs and the second one is over all lattice sites. The time dependent external magnetic field is given by

$$
H(t)=H_{0} \cos (\omega t)
$$

where $t$ is the time and $H_{0}$ is the amplitude of the oscillating magnetic field with a frequency $\omega$. The dynamical evolution of the system may be given by Glauber dynamics 31] based on a master equation

$$
\tau \frac{d}{d t}\left\langle\left\langle c_{i} s_{i}\right\rangle\right\rangle_{r}=-\left\langle\left\langle c_{i} s_{i}\right\rangle\right\rangle_{r}+\left\langle\left\langle c_{i} \tanh \left[\beta c_{i}\left(E_{i}+H(t)\right)\right]\right\rangle\right\rangle_{r},
$$

where $1 / \tau$ is the transition per unit time in a Glauber type stochastic process, $\beta=1 / k_{B} T$ and $k_{B}$ represents the Boltzmann constant, $T$ is the temperature and $E_{i}$ is the local field acting on the site $i$ and it is given by

$$
E_{i}=J \sum_{\delta=1}^{z} c_{\delta} s_{\delta},
$$

where $z$ is the coordination number of the lattice. The inner average brackets in Eq. (3) stands for the thermal average and the outer one (which has a subscript $r$ ) represents the random configurational average which is necessary for including the site dilution effects. 
In order to handle the second term on the right hand side of the Eq. (3) one can use the differential operator technique [23, 24]. By using the differential operator technique, Eq. (3) gets the form

$$
\tau \frac{d}{d t}\left\langle\left\langle c_{i} s_{i}\right\rangle\right\rangle_{r}=-\left\langle\left\langle c_{i} s_{i}\right\rangle\right\rangle_{r}+\left\langle\left\langle c_{i} \exp \left(c_{i} E_{i} \nabla\right)\right\rangle\right\rangle_{r} f(x)
$$

where $\nabla=\partial / \partial x$ is the differential operator and the function $f(x)$ is given by

$$
f(x)=\tanh [\beta(x+H(t))] .
$$

The effect of the differential operator on a function $f(x)$ is given by

$$
\exp (a \nabla) f(x)=f(x+a),
$$

with any constant $a$. By taking into account the two possible values of $c_{i}$ as $c_{i}=0,1$ we can write the exponential term as

$$
\exp \left(a c_{i}\right)=c_{i} \exp (a)+1-c_{i}
$$

where $a$ is any constant. By using Eq. (8) in Eq. (5) we can obtain

$$
\tau \frac{d}{d t}\left\langle\left\langle c_{i} s_{i}\right\rangle\right\rangle_{r}=-\left\langle\left\langle c_{i} s_{i}\right\rangle\right\rangle_{r}+\left\langle\left\langle c_{i} \exp \left(E_{i} \nabla\right)\right\rangle_{r} f(x)\right.
$$

where for site occupations $c_{i}^{2}=c_{i}$ was used. By using Eq. (4) in Eq. (9) we get

$$
\tau \frac{d}{d t}\left\langle\left\langle c_{i} s_{i}\right\rangle\right\rangle_{r}=-\left\langle\left\langle c_{i} s_{i}\right\rangle\right\rangle_{r}+\left\langle\left\langle c_{i} \prod_{\delta=1}^{z}\left[c_{\delta} \exp \left(J s_{\delta} \nabla\right)+1-c_{\delta}\right]\right\rangle\right\rangle_{r} f(x) .
$$

In order to get a polynomial form of the second term on the right hand side of Eq. (10), we write the exponential term in terms of the hyperbolic trigonometric functions

$$
\begin{aligned}
& \tau \frac{d}{d t}\left\langle\left\langle c_{i} s_{i}\right\rangle\right\rangle_{r}=-\left\langle\left\langle c_{i} s_{i}\right\rangle\right\rangle_{r} \\
& +\left\langle\left\langle c_{i} \prod_{\delta=1}^{z}\left[c_{\delta} \cosh (J \nabla)+c_{\delta} s_{\delta} \sinh (J \nabla)+1-c_{\delta}\right]\right\rangle\right\rangle_{r} f(x) .
\end{aligned}
$$

When the product in Eq. (11) is expanded, the multi site spin correlations appear. For simplicity let us handle these correlations with an improved decoupling approximation (DA) [32] as

$$
\left\langle\left\langle c_{i} \ldots c_{j} c_{k} s_{k} \ldots c_{l} s_{l}\right\rangle\right\rangle_{r}=\left\langle\left\langle c_{i}\right\rangle\right\rangle_{r} \ldots\left\langle\left\langle c_{j}\right\rangle\right\rangle_{r}\left\langle\left\langle c_{k} s_{k}\right\rangle\right\rangle_{r} \ldots\left\langle\left\langle c_{l} s_{l}\right\rangle\right\rangle_{r}
$$

with

$$
\left\langle c_{i}\right\rangle_{r}=c, \quad\left\langle\left\langle c_{i} s_{i}\right\rangle\right\rangle_{r}=m .
$$

Detailed discussion of DA in site dilution problem for the static case can be found in 32$]$. By using Eqs. (12) and (13) in Eq. 11 we get

$$
\tau \frac{d m}{d t}=-m+c\left\langle\left\langle[c \cosh (J \nabla)+m \sinh (J \nabla)+1-c]^{z}\right\rangle\right\rangle_{r} f(x),
$$

By using the binomial expansion and writing the hyperbolic trigonometric functions in terms of the exponential functions we get the compact form of Eq. (14) as

$$
\frac{d m}{d t}=\frac{1}{\tau}\left(-m+\sum_{r=0}^{z} A_{r} m_{r}\right)
$$


where

$$
A_{r}=\sum_{p=r}^{z}\left(\begin{array}{c}
z \\
p
\end{array}\right)\left(\begin{array}{c}
p \\
r
\end{array}\right) c^{p-r+1}(1-c)^{z-p} \cosh ^{p-r}(J \nabla) \sinh ^{r}(J \nabla) f(x) .
$$

Eq. (15) can be solved by various numerical methods. In this work we prefer to use the fourth order Runge-Kutta method (RK4) by regarding Eq. (15) as an initial value problem. We can mention that the differential equation derived in Eq. (15) extends up to the term $m^{z}$. Each term in Eq. (15) makes contribution to the solution, because the value of $m$, which is calculated at each time step, is related to the previous $m$ value however the situation is different from the behavior of the equilibrium systems at which high ordered terms can be neglected in the neighborhood of phase transition point.

The system has three Hamiltonian parameters, namely magnetic field frequency $(\omega)$, magnetic field amplitude $\left(H_{0}\right)$ and site concentration $(c)$. For selected values of these Hamiltonian parameters and temperature, RK4 will give convergency behavior after some iterations i.e. the solutions have property $m(t)=m(t+2 \pi / \omega)$ for arbitrary initial value for the magnetization $(m(t=0))$. Thus, after obtaining this convergent region after some transient steps (which depends on Hamiltonian parameters and the temperature) the DOP can be calculated from

$$
Q=\frac{\omega}{2 \pi} \oint m(t) d t
$$

The dynamic nature of the system for a given temperature and Hamiltonian parameters can be determined from the DOP. There are three possible states for the system, namely ferromagnetic $(\mathrm{F})$, paramagnetic $(\mathrm{P})$ and mixed $(\mathrm{F}+\mathrm{P})$. The solution $m(t)$ in the convergent region satisfies

$$
m(t)=-m(t+\pi / \omega),
$$

in the $\mathrm{P}$ phase which is called the symmetric solution. The solution corresponding to $\mathrm{P}$ phase follows the external magnetic field and oscillates around zero value which means that the DOP $Q$ is zero. In the F phase, the solution does not satisfy Eq. (18) and this solution is called as non-symmetric solution which oscillates around a non-zero magnetization value, and does not follow the external magnetic field i.e. the value of $Q$ is different from zero. In these two cases, the observed behavior of the magnetization is regardless of the choice of the initial value of magnetization $m(0)$ whereas the last phase has magnetization solutions symmetric or non-symmetric depending on the choice of the initial value of magnetization corresponding to the coexistence region where $\mathrm{F}$ and $\mathrm{P}$ phases overlap.

\section{Results and Discussion}

In order to determine the effect of the Hamiltonian parameters on the critical temperature of the system we present the phase diagrams for a honeycomb lattice $(z=3)$ in $\left(k_{B} T_{c} / J-H_{0} / J\right)$ and $\left(k_{B} T_{c} / J-c\right)$ planes with frequencies $\omega=0.1,0.5,1.0,5.0$ in Figs. (4) and (4), respectively. 
At first sight we can see from Fig. (4) that as $c$ value decreases then the $F$ phase region gets narrower in the $\left(k_{B} T_{c} / J-H_{0} / J\right)$ plane. This is an expected result, since as the lattice sites are diluted more and more then the energy contribution which comes from the spin-spin interactions gets smaller. Hence, the system can undergo a DPT at smaller critical temperatures, due to the energy originating from the temperature and (or) magnetic field which overcomes the spin-spin interactions. Therefore, the ferromagnetic regions in $\left(k_{B} T_{c} / J-H_{0} / J\right)$ plane get narrower. In addition, increasing $H_{0} / J$ values reduce the dynamical critical temperature while as $\omega$ increases then the $F$ phase region gets wider in the $\left(k_{B} T_{c} / J-H_{0} / J\right)$ plane for all concentration values. These observations can also be explained by the well known mechanisms underlying the DPT's. In other words, increasing field amplitude facilitates the phase transition due to the increasing energy coming from the oscillating magnetic field which tends to align the spins in its direction whereas an increasing field frequency gives rise to a growing phase delay between the magnetization and magnetic field (i.e. the magnetization cannot follow the oscillating magnetic field) and this makes the occurrence of the DPT difficult, as a result of this mechanism critical temperature increases. Moreover, the low concentration phase diagrams in Fig. (4), which appear at small $\omega$ values such as $w=0.1$ and 0.5 , exhibit a reentrant behavior and the reentrance disappears at a certain value of increasing frequency between $0.5<\omega<1$.0. For example, as seen from the curve with $H_{0} / J=0.2, \omega=0.5$ and $c=0.6$, corresponding to the upper right panel Fig. (44), as the temperature increases starting from a value in the paramagnetic phase then the system undergoes a second order phase transition due to the thermal agitations and remains at a ferromagnetic order for a while. As the temperature increases further then the system undergoes another second order phase transition from a ferromagnetic to a paramagnetic phase.

An interesting result is that the $F+P$ region disappears in the $\left(k_{B} T_{c} / J-H_{0} / J\right)$ plane for sufficiently weak dilution. In order to focus on this interesting behavior, we depict the phase diagrams in the same plane for $\omega=1.0$ with the concentration values $c=1.00,0.98,0.96,0.94,0.92$ in Fig. (44). As seen in Fig. (44), as the concentration value decreases then $F+P$ region which appears at low temperatures and high field amplitude values, gradually shrinks then disappears at a certain concentration value. Then we can not see this region in the phase diagrams which are shown in Fig. (4) except the concentration value $c=1.0$. On the other hand, one observes a dynamic tricritical point only in a narrow range of site concentration $c$ which disappears at a certain concentration value. Namely, as seen in Fig. (44) for $c=0.94$ we observe a reentrant behavior of first order instead of the coexistence region while for $c=0.92$ the first order reentrance turns into a second order reentrance.

In order to probe the concentration dependence of the dynamic nature of the system, we plot the phase diagrams in $\left(k_{B} T_{c} / J-c\right)$ plane for some selected values of Hamiltonian parameters in Fig. (4). We can see that the results shown in Fig. (44) are completely consistent with the results presented in Fig. (44). As the concentration $c$ of magnetic atoms decreases then the ferromagnetic region gets narrower, as expected. In Fig. (4), 
we can also observe that there are mainly two reentrance regions which are located for $H_{0} / J=0.2$ with $\omega=0.1,0.5$ and for $H_{0} / J=1.2,1.4$ with $\omega=0.5,1.0,5.0$, respectively. The former is nothing but just the reentrant region shown in the upper panels in Fig. (44) that observed for low concentration values. By taking into account these observations, we can conclude that the system may exhibit reentrant behavior for a wide range of Hamiltonian parameters as a result of a competition between the interactions that exist in the system. Hence, in the paramagnetic phase, the increasing temperature inhibits the ability of response of the magnetization to the external field even at low frequency region by allowing a transition from paramagnetic phase to ferromagnetic one.

At a certain $c$ value the system undergoes a phase transition at zero temperature which means that the system cannot exhibit an ordered phase below this certain $c$ value for the phase diagrams in $\left(k_{B} T_{c} / J-c\right)$ plane. No matter the phase diagrams in $\left(k_{B} T_{c} / J-c\right)$ plane shows reentrance or not, for concentration values lower than this certain concentration value, namely site percolation threshold value $\left(c^{\star}\right)$, the system cannot exhibit an ordered phase at all. In other words, for $c>c^{\star}$ the system forms an infinite cluster of lattice sites. However, as $c$ gets closer to $c^{\star}$ then isolated finite clusters appear, since the spin-spin interaction energy become insufficient to compete with the magnetic field energy, even in the absence of the thermal energy (i.e. at zero temperature). As a result of this, the system cannot exhibit long range ferromagnetic order even at zero temperature for $c<c^{\star}$. The variation of $c^{\star}$ as a function of the magnetic field amplitude for frequencies $\omega=0.1,0.5,1.0$ and 5.0 can be seen in Fig. (4). As seen in Fig. (44), $H_{0} / J$ and $\omega$ parameters have an opposite effect on $c^{\star}$. We can say that the general effect of increasing $H_{0} / J$ is to increase the value of $c^{\star}$ whereas increasing value of $\omega$ parameter decreases the value of $c^{\star}$. An important observation in Fig. (41) is the appearance of plateaus for different $\omega$ values. For $\omega=0.1, c^{\star}$ value is observed between $0.5<H_{0} / J<1.0$ which almost does not change its shape with varying $H_{0} / J$ values. As $\omega$ value gets higher (e.g $\omega=5.0$ curve on left panel in Fig. (4)) then another wider plateau originates within the range $0.0<H_{0} / J<1.0$. We can see from the right panel in Fig. (4) that the same situation holds for a square lattice $(z=4)$. We should state that in the absence of magnetic field $\left(H_{0} / J=0\right), c^{\star}$ values are exactly the same values with the static case obtained by the same approximation (DA) [33].

It is well known that one cannot write an appropriate expression for the free energy including the presence of the time dependent oscillating magnetic field. Hence, we cannot use the free energy expression to determine the type of the phase transition (first or second order). Hence, we simply follow a procedure described briefly below. Since the time averaged magnetization over a full cycle of the external magnetic field acts as the DOP then we may check the temperature dependence of it. Strictly speaking, if the DOP decreases continuously to zero in the vicinity of critical temperature, this transition is classified as of the second order whereas if it vanishes discontinuously then the transition is assumed to be of the first order. The results of the previously published works have pointed out that all transitions from $F$ or $P$ phases to $F+P$ phase (or vice 
versa) are of the first order transitions [25, 26]. Hence we shall only investigate the transitions between $F$ and $P$ phases, especially for the Hamiltonian parameters which exhibit reentrance. The variation of the DOP with the temperature for some selected values of the Hamiltonian parameters can be seen in Fig. (4). From the left panel in Fig. (4), we can see that all transitions on the $c=0.6$ phase diagram with $\omega=0.5$ corresponding to the case in the upper right panel in Fig. (44) are of the second order. The situation is similar for the middle panel of Fig. (4) where $H_{0} / J=0.2$ and $\omega=0.1$ corresponding to the upper right panel in Fig. (4). Finally, we can conclude with the transitions shown in the phase diagrams with $\omega=1.0$ and $H_{0} / J=1.4$ which are of the second order as seen in the right panel of Fig. (4). A detailed investigation for the variation of the DOP with the temperature for other Hamiltonian parameters shows us that all phase transitions of the site diluted system are of the second order except the transitions from $F$ or $P$ phases to $F+P$ phase which occurs at low temperature and high field amplitude values and within a very narrow region of the Hamiltonian parameter values (e.g. for $\omega=1.0, c=0.94$ curve as shown in Fig.(低)).

\section{Conclusion}

In this work, we have investigated the dynamic nature of the critical phenomena which is observed for a site diluted Ising ferromagnet defined on a honeycomb lattice $(z=3)$ driven by an external oscillating magnetic field by means of EFT. We have used a Glauber-type stochastic process to describe the time evolution of the system. We have given the global phase diagrams, including the reentrant phase transitions. According to our calculations, $\mathrm{F}+\mathrm{P}$ phase disappears for sufficiently weak dilution of lattice sites.

EFT method takes the standard mean field predictions one step forward by taking into account the single site correlations which means that the thermal fluctuations are partially considered within the framework of EFT. Although all of the observations reported in this work shows that EFT can be successfully applied to such nonequilibrium systems in the presence of quenched site disorder, the true nature of the physical facts underlying the observations displayed in the present work (especially the origin of the coexistence phase) may be further understood with an improved version of the present EFT formalism which can be achieved by attempting to consider the multi site correlations which originate when expanding the spin identities. We believe that this attempt could provide a treatment beyond the present approximation.

In conclusion, we hope that the results obtained in this work would shed light on the further investigations of the dynamic nature of the critical phenomena in disordered systems and would be beneficial from both theoretical and experimental points of view.

\section{References}

[1] Chakrabarti B K and Acharyya M 1999 Rev. Mod. Phys. 71847

[2] Tome T and de Oliveira M J 1990 Phys. Rev. A 414251

[3] Jiang Q, Yang H N and Wang G C 1995 Phys. Rev. B 5214911 
[4] Robb D T, Xu Y H, Helling O, McCord J, Berger A, Novotny M A, Rikvold P A 2008 Phys. Rev. B 78134422

[5] Kanuga K and Cakmak M 2007 Polymer 487176

[6] Acharyya M 1998 Phys. Rev. E 58179

[7] Zimmer M F 1993 Phys. Rev. E 473950

[8] Luse C N and Zangwill A 1994 Phys. Rev. E 50224

[9] Acharyya M 1997 Phys. Rev. E 562407

[10] Han Z, Shuai D and Liu J -M 2004 Phys. Rev. B 70132403

[11] Acharyya M 1999 Phys. Rev. E 59218

[12] Acharyya M and Chakrabarti B K 1995 Phys. Rev. B 526550

[13] Acharyya M 1999 Phys. Rev. E 59218

[14] Sides S W, Rikvold P A and Novotny M A 1998 Phys. Rev. Lett. 81834

[15] Sides S W, Rikvold P A and Novotny M A 1998 Phys. Rev. E 576512

[16] Sides S W, Rikvold P A and Novotny M A 1999 Phys. Rev. E 592710

[17] Korniss G, Rikvold P A and Novotny M A 2002 Phys. Rev. E 66056127

[18] Keskin M, Canko O and Deviren B 2006 Phys. Rev. E 74011110

[19] Keskin M, Canko O and Temizer U 2005 Phys. Rev. E 72036125

[20] Buendia G M and Machado E 1998 Phys. Rev. E 581260

[21] Keskin M, Kantar E and Canko O 2008 Phys. Rev. E 77051130

[22] Acharyya M, Chakrabarti B K and Stinchcombe R B 1994 J. Phys. A 271533

[23] Honmura R and Kaneyoshi T 1979 J. Phys. C 123979

[24] Kaneyoshi T 1993 Acta Phys. Pol. A 83703

[25] Deviren B, Canko O and Keskin M 2010 Chin. Phys. B 19050518

[26] Kinoshita T, Fujiyama B, Tokita M and Idogaki T 2009 Journal of Physics: Conference Series 150042091

[27] Xiao-Ling S and Guo-Zhu W 2009 Commun. Theor. Phys. 51927

[28] Shi X, Wei G and Li L 2008 Phys. Lett. A 3725922

[29] Xiao-Ling S, Li W and Guo-Zhu W 2011 Commun. Theor. Phys. 55715

[30] Shi X and Wei G 2010 Phys. Lett. A 3741885

[31] Glauber R J 1963 J. Math. Phys. 4294

[32] Tucker J W 1991 J. Magn. Magn. Mater. 102144

[33] Saber M 1997 Chin. Jour. Phys. 35577 


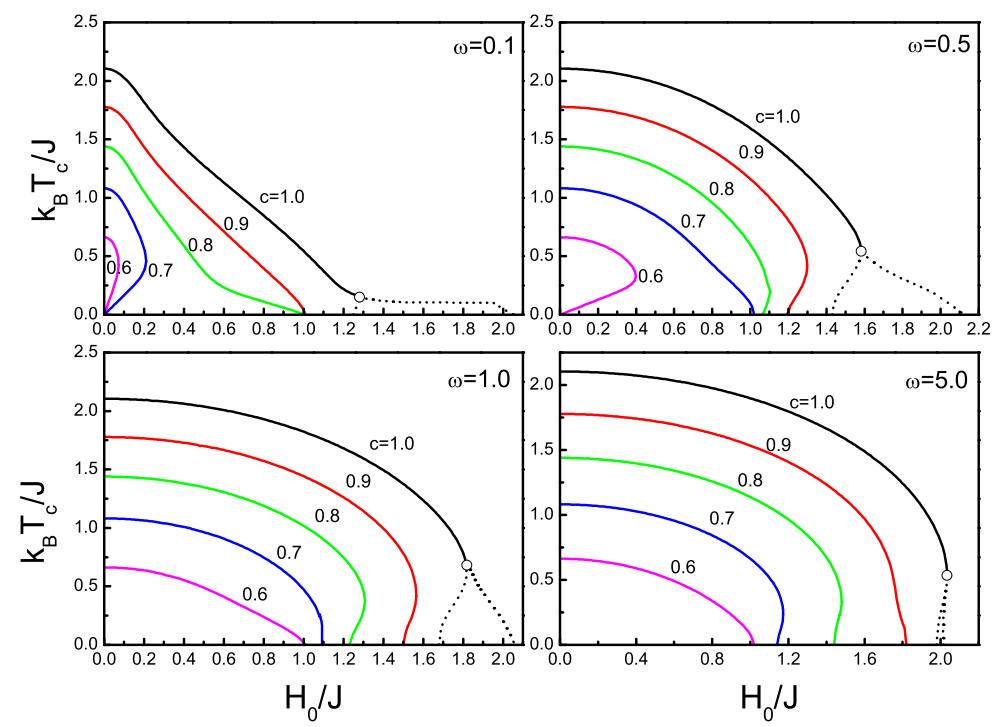

Figure 1. Phase diagrams in $\left(k_{B} T_{c} / J-H_{0} / J\right)$ plane for a honeycomb lattice with some selected values of $\omega$ and $c$. The solid (dotted) lines correspond to the second (first) order transitions and solid symbols denote the dynamic tricritical points.

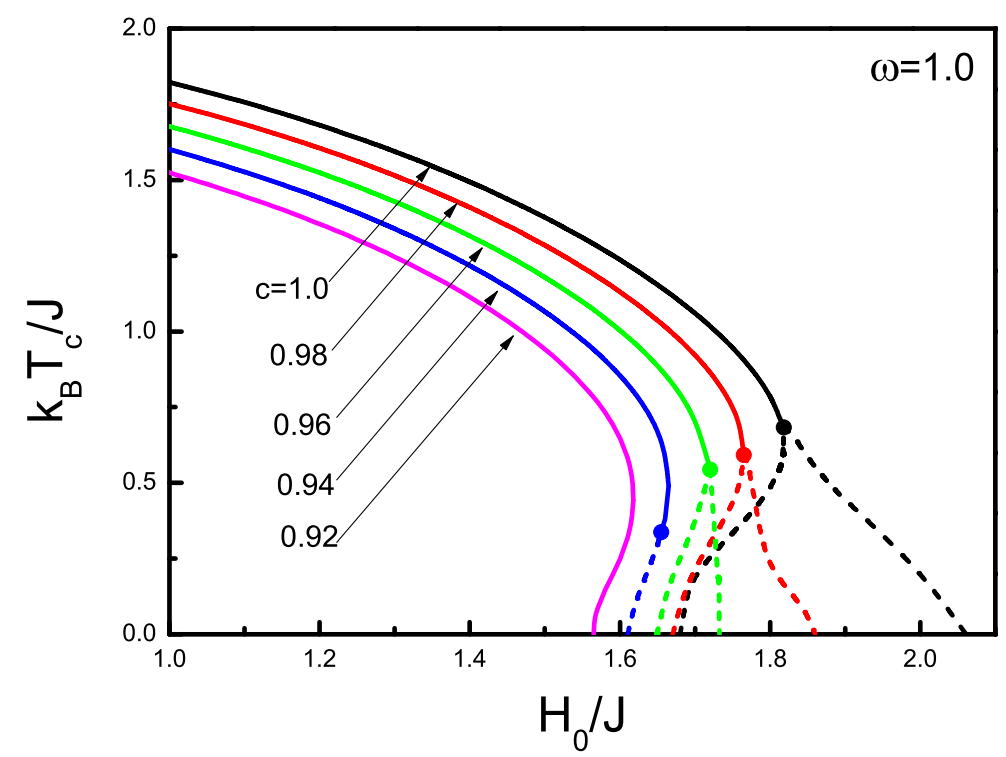

Figure 2. Phase diagrams for a honeycomb lattice in $\left(k_{B} T_{c} / J-H_{0} / J\right)$ plane for $\omega=1.0$. The numbers accompanying each curve denote the value of site concentration c. The solid (dashed) curves correspond to the second (first) order transitions and solid symbols denote the dynamic tricritical points. 


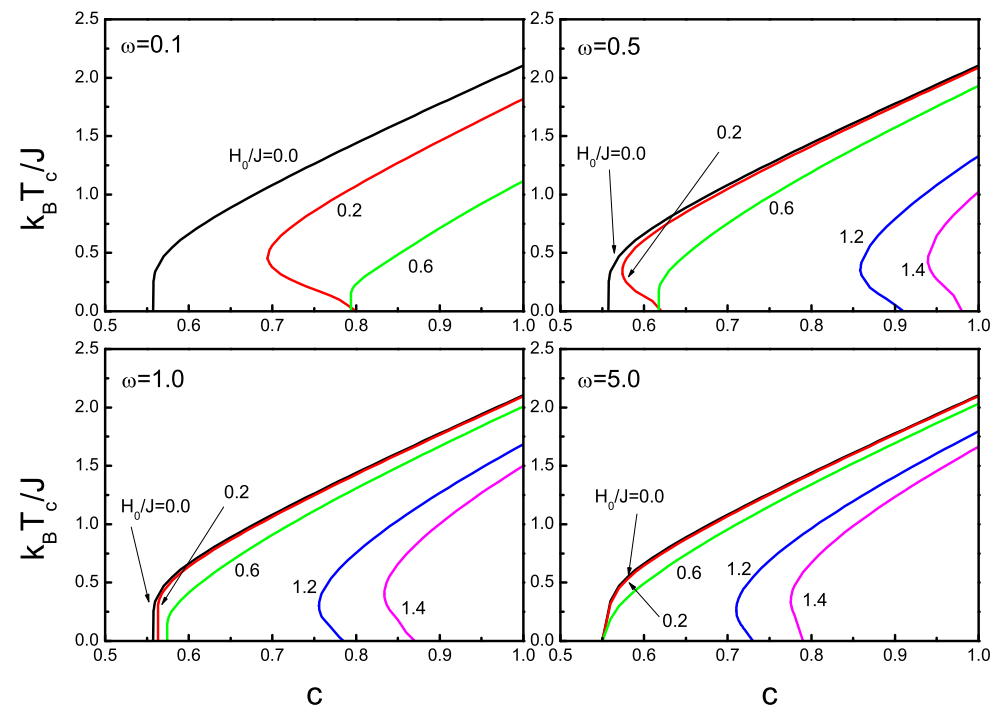

Figure 3. Phase diagrams for a honeycomb lattice in $\left(k_{B} T_{c} / J-c\right)$ plane for some selected values of $\omega$ and $H_{0} / J$. The numbers accompanying each curve denote the value of the amplitude $H_{0} / J$ of the oscillating magnetic field.
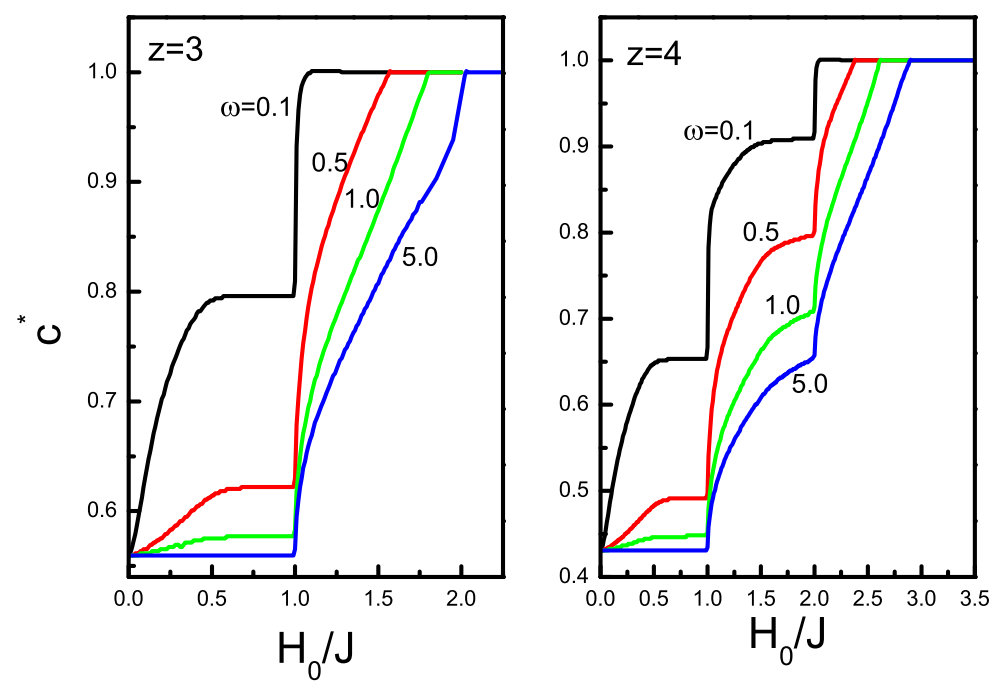

Figure 4. Variation of the site percolation threshold $c^{\star}$ of honeycomb and square lattices with the amplitude $H_{0} / J$ of the oscillating magnetic field. The numbers accompanying each curve represent the frequency $\omega$ of the oscillating field. 

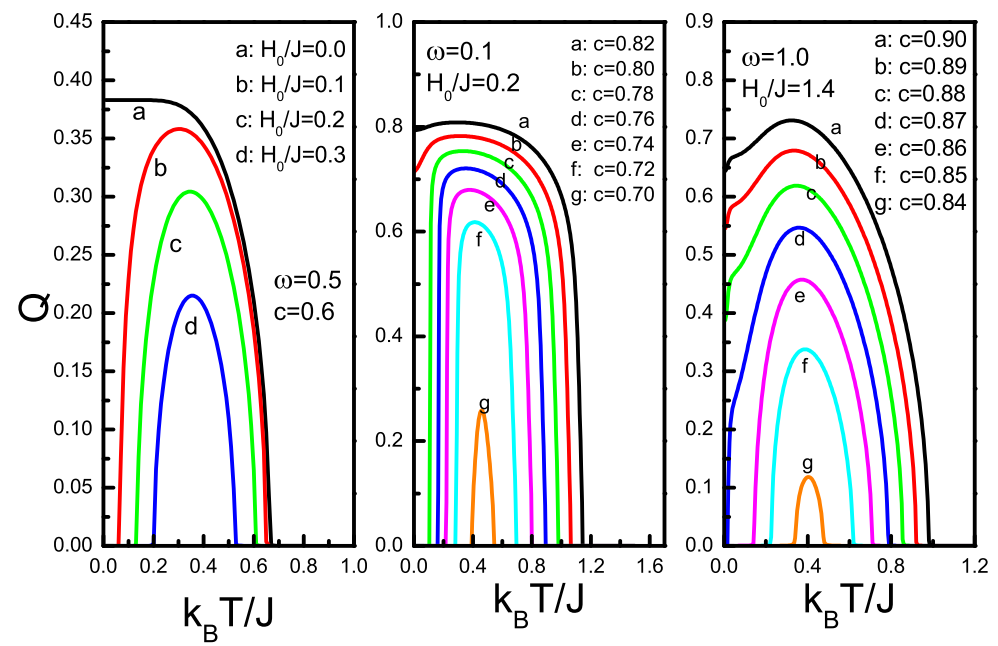

Figure 5. Variation of the DOP $Q$ for a honeycomb lattice as a function of the temperature for some selected values of the Hamiltonian parameters $\omega, H_{0} / J$ and $c$. 